\title{
Fairness in Power Flow Network Congestion Management with Outer Matching and Principal Notions of Fair Division
}

\author{
Brinn Hekkelman \\ Centrum Wiskunde \& Informatica (CWI) and TU Delft \\ Amsterdam, The Netherlands \\ B.Hekkelman@cwi.nl
}

\begin{abstract}
The problem of network flow congestion occurring in power networks is increasing in severity. Especially in low-voltage networks this is a novel development. The congestion is caused for a large part by distributed and renewable energy sources introducing a complex blend of prosumers to the network. Since congestion management solutions may require individual prosumers to alter their prosumption, the concept of fairness has become a crucial topic of attention. This paper presents a concept of fairness for low-voltage networks that prioritizes local, outer matching and allocates grid access through fair division of available capacity. Specifically, this paper discusses three distinct principal notions of fair division; proportional, egalitarian, and nondiscriminatory division. In addition, this paper devises an efficient algorithmic mechanism that computes such fair allocations in limited computational time, and proves that only egalitarian division results in incentive compatibility of the mechanism.
\end{abstract}

\section{CCS CONCEPTS}

- Networks $\rightarrow$ Network resources allocation; • Hardware $\rightarrow$ Smart grid; • Computing methodologies $\rightarrow$ Multi-agent systems. KEYWORDS

fairness, fair division, energy, smart grid, flow networks, congestion management, local matching, resource allocation

\section{ACM Reference Format:}

Brinn Hekkelman and Han La Poutré. 2020. Fairness in Power Flow Network Congestion Management with Outer Matching and Principal Notions of Fair Division. In The Eleventh ACM International Conference on Future Energy Systems (e-Energy'20), June 22-26, 2020, Virtual Event, Australia. ACM, New York, NY, USA, 10 pages. https://doi.org/10.1145/3396851.3397701

\section{INTRODUCTION}

With the increasing share of renewable energy sources comes an increase in electrical grid congestion [26]. The consequences of this rapid increase in congestion are already seen in soaring congestion management costs [18], even at the low- and medium-voltage levels. Current grid congestion management is insufficiently prepared

Permission to make digital or hard copies of all or part of this work for personal or classroom use is granted without fee provided that copies are not made or distributed for profit or commercial advantage and that copies bear this notice and the full citation on the first page. Copyrights for components of this work owned by others than the author(s) must be honored. Abstracting with credit is permitted. To copy otherwise, or republish, to post on servers or to redistribute to lists, requires prior specific permission and/or a fee. Request permissions from permissions@acm.org.

() Brinn Hekkelman and Han La Poutré 2020. This is the author's version of the work. It is posted here for your personal use. Not for redistribution. The difinitive version was published in ACM e-Energy'20, June 22-26, 2020, Virtual Event, Australia. https://doi.org/10.1145/3396851.3397701

\author{
Han La Poutré \\ Centrum Wiskunde \& Informatica (CWI) and TU Delft \\ Amsterdam, The Netherlands \\ Han.La.Poutre@cwi.nl
}

for the changes in electrical grid operation brought about by the energy transition. New solutions for congestion management come in various forms $[12,20,28]$, often focusing on the introduction of electric vehicles (EVs) [14, 17, 21, 22] or the decentralized, multiagent aspect of distributed energy resources (DERs) [6, 25, 27].

However, these solutions typically do not explicitly take into account a concept of fairness. Meanwhile, the energy transition is bringing about a paradigm shift from 'supply follows demand' to 'demand follows supply' that, as a consequence, places the responsibility for congestion increasingly on individual prosumers. This development makes it crucial to explicitly incorporate concepts of fairness in congestion management solutions [13]. The European Commission emphasizes the importance of fairness in energy, stating that "energy is a critical good, absolutely essential for full participation in modern society. The clean energy transition also needs to be fair for those sectors, regions or vulnerable parts of society affected by the energy transition." [9]

In this paper we lay a theoretical foundation for fair congestion management, using a congestion model similar to those used in $[4,15,21,26]$. We propose an algorithmic mechanism of low computational complexity that combines a locally oriented novel fairness concept with principal notions of fair division. We prove that this algorithmic mechanism divides the available network capacity maximally over the prosumers.

Specifically, in this paper we propose local, outer matching as a novel concept of fairness for congestion management in lowvoltage networks. This concept of fairness requires that congestion is resolved with recursive matching of supply and demand in localities outward from nodes in the network. Local, outer matching thus prioritizes matching in the peripheral of the network, reducing strain and losses on the network infrastructure.

Still, when congestion occurs, the available network capacity must be fairly divided over the affected prosumers. The fair division of goods and fairness in general are established and active fields of research in mathematics and economics [2, 3, 5, 8, 19, 24]. One application domain is that of communications networks, where network capacity must be fairly divided over users [11]. While congestion management in power flow networks faces similar fair division problems, power flow networks are concerned with a singlecommodity flow as opposed to peer-to-peer data transmission. In the energy domain currently, fairness is considered mostly for EV charging [7] and DER related pricing [16].

In this paper we discuss three principal notions of fair division that may be combined with the novel fairness concept of local, outer matching to perform its division. The principal notions of fair division we discuss here are: proportional, first proposed by Steinhaus [23] and sometimes referred to as 'simple fair division'; 
egalitarian, which is closely related to the concept of envy-freeness first proposed by Gamow and Stern [10]; and nondiscriminatory, which is a natural counterpart to the egalitarian notion of fair division.

It is apparent that fairness in power flow networks has a combination of aspects. On the one hand, fair division of network capacity is required. On the other hand, the single-commodity flow necessitates supply-demand matching throughout the network. Matching supply and demand locally is a newly accepted paradigm for energy networks that stimulates the use of local infrastructure. This introduction of localities affects concepts of fairness [1]. Envisioned autarkic-like local communities and neighbourhoods thus demand a local approach to congestion management and concepts of fairness.

To this end, we devise an algorithmic mechanism that computes the combination of local, outer matching with the discussed principal notions of fair division, resulting in locally oriented congestion solutions that make maximal use of the network capacity. We then prove that the egalitarian notion of fairness results in an incentive compatible mechanism, while the proportional and nondiscriminatory notions of fairness do not result in an incentive compatible mechanism. Finally, we show that the proposed algorithmic mechanism computes congestion solutions in limited computational time, which is essential for application in the energy domain.

The contributions of this paper to the state of the art can be summarised as follows:

- We propose a novel concept of fairness for congestion management called local, outer matching.

- We discuss the principal notions of proportional, egalitarian, and nondiscriminatory fair division that we combine with the concept of local, outer matching.

- We devise an algorithmic mechanism that combines local, outer matching with notions of fair division to compute maximal congestion solutions in limited computational time.

- We prove that this mechanism is incentive compatible when using the egalitarian notion of fair division, and is not incentive compatible when using the proportional or nondiscriminatory notions of fair division.

The paper is organized as follows. First, Sections 2 and 3 introduce the setting, model, and useful concepts. Section 4 then formally defines division and discusses the three principal notions of fair division. Section 5 defines the novel fairness concept of local, outer matching and provides congestion solutions that are proven to be local, outer matchings that make maximal use of network capacity. Finally, Section 6 presents the algorithmic mechanism that combines local, outer matching with the principal notions of fair division. The incentive compatibility results follow in Section 7 and the computational complexity results in Section 8. Section 9 concludes the paper.

\section{SETTING}

We consider an electrical power flow network that consists of prosumers connected to each other by electrical grid lines. These grid lines have a maximum reliable capacity (which is usually less than their physical or thermal limit). Grid congestion occurs when electrical power flow caused by the prosumers exceeds some line capacities. This means that it is not always possible to realise the desired prosumption of all prosumers within the network constraints. Congestion management is the practice of reducing, resolving, or preventing grid congestion by deviating from the desired prosumptions in order to accommodate the network constraints.

We follow a modelling approach similar to those in $[4,15,21,26]$ that lay the theoretical foundations for congestion management, e.g. by focusing on active power curtailment. As such, we model an electrical power flow network as a tree (representing almost all real-world low-voltage networks), the line capacities as edge weights, the prosumers as agents that are either consumers or producers located at the vertices, and the desired prosumptions as agent desires. Low-voltage networks are usually connected to larger electrical grids through a substation. This connection to an external grid may be modelled as a virtual edge, which will also have a line capacity modelled as an edge weight.

\section{MODEL}

Let $T=(V, E)$ be a rooted weighted tree. Let a virtual edge at the root $r$ represent the connection to a virtual parent that represents an external network. Let the edge weights be positive, representing flow capacities. Denote the weight of an edge between vertex $i \in V$ and its parent by $C_{i}$. In addition, consider a set of agents $A$ distributed over the vertices $V$. Finally, for each agent $a \in A$, consider its desire $d_{a}$. A positive desire indicates a consumer while a negative desire indicates a producer. Let $Q, P \subset A$ be the sets of consumers and producers, respectively.

Definition 3.1 (Congestion Tree). Define a congestion tree $T=$ $(V, E, A)$ as a tree $T=(V, E)$ with $\operatorname{root} r$, edge weights $C_{i}$ for $i \in V$, and agents $a \in A$ with desires $d_{a}$ located at the vertices $i \in V$.

The subtree of a congestion tree $T=(V, E, A)$ is again a congestion tree, and is denoted by $T_{i}=\left(V_{i}, E_{i}, A_{i}\right)$ where $i$ is its root. A subtree $T_{i}=\left(V_{i}, E_{i}, A_{i}\right)$ inherits the edge weights of $T=(V, E, A)$, with its virtual edge inheriting the weight of the edge between $i$ and its parent in $T=(V, E, A)$. See Figure 1 for a representation.

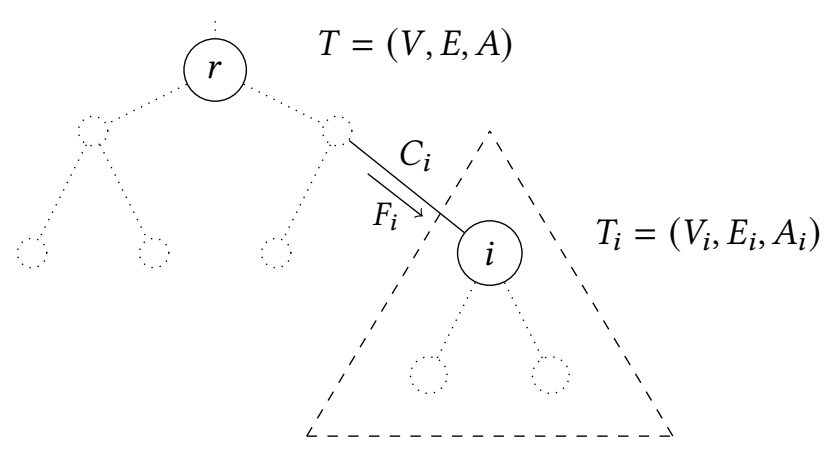

Figure 1: A representation of a congestion tree $T=(V, E, A)$, highlighting the situation around a vertex $i$.

\subsection{Congestion Management}

Congestion management is the practice of reducing, resolving, or preventing congestion in a network, and can take various forms. In 
graph theory, flow networks consider flow resulting from a single source and a single sink in a graph with flow capacities on the edges. Power networks usually deal with a more complex situation where many distributed prosumers are participating in a market.

In the context of congestion trees, congestion management is performed by allocating network access to agents based on their desires and the network constraints. Such allocations could be strictly enforced or used as reference for penalties or incentives.

Definition 3.2. An allocation $Y$ on a congestion tree $T=(V, E, A)$ is a map $Y: A \rightarrow \mathbb{R}$.

Notation. For $B \subseteq A$, abbreviate $\sum_{a \in B} Y(a)$ as $Y(B)$.

When agents that represent prosumers are subject to such an allocation, the resulting prosumptions lead to (electrical power) flows in the network.

Definition 3.3 (Incoming and Locally Balanced Flows). Given an allocation $Y$ on a congestion tree $T=(V, E, A)$, define the incoming flow $F_{i}(Y)$ and the locally balanced flow $L B F_{i}(Y)$ of a subtree $T_{i}=$ $\left(V_{i}, E_{i}, A_{i}\right)$ as:

$$
\begin{aligned}
F_{i}(Y) & =Y\left(A_{i}\right)=\sum_{a \in A_{i}} Y(a) \\
L B F_{i}(Y) & =\frac{1}{2}\left[\sum_{a \in A_{i}}|Y(a)|-\left|\sum_{a \in A_{i}} Y(a)\right|\right] .
\end{aligned}
$$

The incoming flow $F_{r}(Y)$ of an allocation $Y$ on a congestion tree $T=(V, E, A)$ with root $r$ thus represents the total amount of electrical power demanded of, or supplied to, the external grid. The incoming flow also gives the flow over each edge, with $F_{i}(Y)$ being the flow to vertex $i$ from its parent. See Figure 1 for a representation.

The locally balanced flow $L B F_{r}(Y)$ represents the total amount of electrical power that flows between the agents within the congestion tree $T=(V, E, A)$ with root $r$, including between agents that share a vertex. The locally balanced flow $L B F_{r}(Y)$ is a measure for the matching of consumer and producer desires within the congestion tree $T=(V, E, A)$.

Definition 3.4 (Desire Compatible). An allocation $Y$ on a congestion tree $T=(V, E, A)$ is desire compatible if

$$
0 \leq Y(a) \leq d_{a} \quad \text { or } \quad 0 \geq Y(a) \geq d_{a} \quad \forall a \in A .
$$

Definition 3.5 (Congestion Free). An allocation $Y$ on a congestion tree $T=(V, E, A)$ is congestion free if, for each vertex $i \in V$, the incoming flow $F_{i}(Y)$ of the subtree $T_{i}=\left(V_{i}, E_{i}, A_{i}\right)$ does not exceed the flow capacity $C_{i}$ of its virtual edge:

$$
\left|F_{i}(Y)\right| \leq C_{i} \quad \forall i \in V .
$$

Definition 3.6 (Feasible). An allocation $Y$ on a congestion tree is feasible if it is both desire compatible and congestion free.

The set of feasible allocations forms the solution space for the problem of congestion management. Within this solution space, some allocations are more desirable than others because they make better use of the available network capacity.

Definition 3.7 (Base Allocation). A feasible allocation $Y$ on a congestion tree $T=(V, E, A)$ with root $r$ is a base allocation if it maximizes the locally balanced flow $\operatorname{LBF}_{r}(Y)$ and has incoming flow $F_{r}(Y)=0$.
A base allocation maximally matches consumer and producer desires in a congestion tree, without interacting with the external grid it is connected to.

Definition 3.8 (Max Allocation). A feasible allocation $Y$ on a congestion tree $T=(V, E, A)$ with root $r$ is a max allocation if it maximizes $\left|F_{r}(Y)\right|$ under the condition that the locally balanced flow $\operatorname{LBF}_{r}(Y)$ is maximal.

A max allocation maximizes the use of the available network capacity by making maximal use of the connection to the external grid after maximally matching consumer and producer desires internally. A max allocation can be viewed as a base allocation plus an allocation of the remaining unmatched desires.

\section{PRINCIPAL NOTIONS OF FAIR DIVISION}

Congestion management leads directly to the question of fair division. The limited available network capacity does not belong to any one agent, and thus it must be fairly divided. The field of fair division of goods considers such division problems. In this case a number of agents lay claim to a portion of a divisible good, the network capacity, but the sum of their claims exceeds the availability of the good. Since energy is a critical good, it is of great importance that the division of capacity, which dictates network access, be fair to all agents. However, the notion of which choice of division constitutes a fair division is subjective.

Consider two agents $a$ and $b$ that have respective claims $d_{a}$ and $d_{b}$ to a quantity $k$ of a divisible good. This situation may be visualized as in Figure 2. The point of the claims $\left(d_{a}, d_{b}\right)$ represents the outcome desired by the agents, while the line intersecting the axes represents the available quantity $k$ of the good.

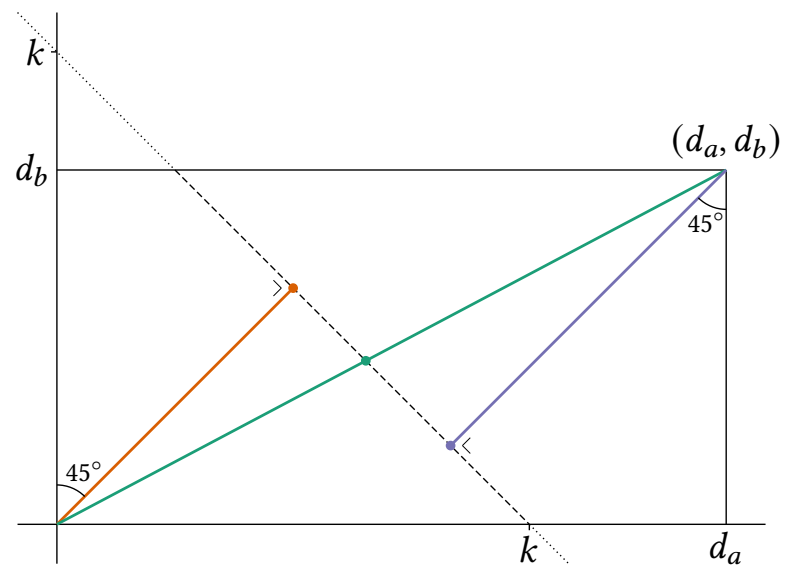

Figure 2: Three principal notions of fair division illustrated: proportional (green, middle), egalitarian (red, left), and nondiscriminatory (blue, right).

The set of divisions is the set of points that exactly divide the quantity $k$ and do not allocate to agents more than their claims. In Figure 2 this is the set of points on the dashed line segment inside the rectangle. If this set is empty because the quantity $k$ is larger than the sum of the claims, then there is no real division problem and the point of claims $\left(d_{a}, d_{b}\right)$ is taken as the division. 
This representation of the division of a good over claims can be extended to any number $n$ of agents by an $n$-dimensional space with an $n-1$ dimensional surface representing the quantity $k$. This leads to the following definition.

Definition 4.1 (Division). The division $\operatorname{Div}(k, Y, A)$ of a positive value $k$ over an allocation $Y$ (of claims) with positive values on a set of agents $A$, is an allocation $D$ on $A$ with

$$
D(A)=\min (k, Y(A))
$$

and

$$
D(a) \in[0, Y(a)] \quad \forall a \in A .
$$

If $k \geq Y(A)$, then $D$ is simply identical to the allocation $Y$ on $A$.

A notion of fair division is the choice of a specific division from the set of divisions. Visually, this means that a notion of fair division is the choice of a specific point on the $n-1$ dimensional plane that represents the quantity $k$, inside the $n$-dimensional hyperrectangle drawn by the origin and the claims. Figure 2 shows three principal notions of fair division:

- The proportional notion of fairness finds the point where the line from the origin to the point of claims intersects the set of divisions. See the green line in Figure 2. Each agent is allocated a portion of the good that is proportional to the ratio of its claim to the sum of all claims. This division treats all agents equally, preserving the relations between the claims.

- The egalitarian notion of fairness finds the point in the set of divisions that is closest to the origin. See the red line in Figure 2. Each agent is allocated the same portion, unless its claim is smaller than that portion. This division treats all agents equally, reducing all claims to the same amount.

- The nondiscriminatory notion of fairness finds the point in the set of divisions that is closest to the point of claims. See the blue line in Figure 2. The portion of each agent is reduced by the same amount regardless of its claim, to a minimum of zero. This division treats all agents equally, reducing all claims by the same amount.

\section{LOCAL, OUTER MATCHING AND FAIRNESS}

The matching of consumer and producer desires is an important aspect of congestion management in congestion trees. On the one hand, the distributed nature of prosumers in electrical power flow networks is the cause of much congestion. On the other hand, the presence of both consumers and producers provides opportunity for mitigating or resolving congestion by locally balancing excessive production or consumption.

Local matching of supply and demand is a newly accepted paradigm for future energy networks. It stimulates the use of local infrastructure as a push towards envisioned autarkic-like local communities and neighbourhoods, reducing strain and losses on the energy network in the process. This local matching of supply and demand also, again, emphasizes the importance of fairness. Factors such as the relative locality of the prosumers will play an important role in concepts of fairness regarding the energy domain.

We address this important problem by presenting local, outer matching as an efficient and fair concept for matching consumer and producer desires. A local, outer matching solution prioritizes local matching in the peripheral where prosumers are furthest away from the substation.

Definition 5.1 (Local, Outer Matching). A feasible allocation $Y$ on a congestion tree $T=(V, E, A)$ is a local, outer matching if the locally balanced flow $L B F_{i}(Y)$ is maximal for each subtree $T_{i}=\left(V_{i}, E_{i}, A_{i}\right)$.

To make maximal use of the available network capacity, a local, outer matching solution is sought that is also a max allocation or a base allocation, depending on the envisioned interaction with the external grid. A max allocation makes maximal use of the external grid as well as the network capacity, while a base allocation is self-balanced and makes maximal use of the network capacity for internally balanced flows. See Figure 3 for some examples. The FairMax and FairBase allocations from Definition 5.2 present such local, outer matching solutions. Their maximal capacity use and local, outer matching are proven by Theorem 5.4.

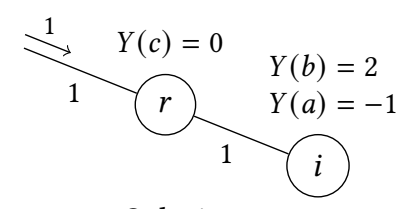

Solution 1

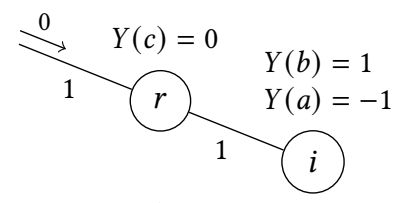

Solution 3

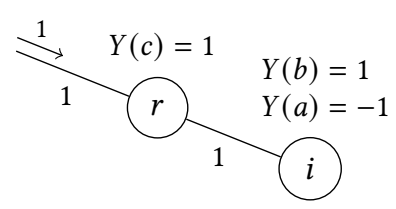

Solution 2

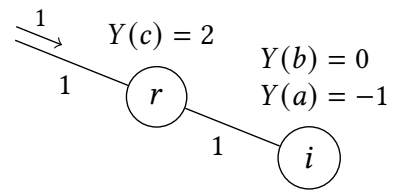

Solution 4
Figure 3: Example congestion solutions for a simple congestion tree $T=(V, E, A)$ consisting of a root $r$ with $C_{r}=1$ containing agent $c$ with $d_{c}=2$, and a second vertex $i$ with $C_{i}=1$ containing both agents $b$ and $a$ with $d_{b}=2$ and $d_{a}=-1$. Solutions 1, 2, and 3 are local, outer matchings. Solutions 1, 2, and 4 are max allocations. Solution 3 is a base allocation.

Let $T=(V, E, A)$ be a congestion tree. Because of the priority given to outer matching from the leaves towards the root, the definitions of local, outer matching allocations are recursive. For a vertex $i \in V$, the consumer and producer desires of all consumers and producers at vertices outward from the vertex $i$ are maximally matched. This results in a chain of matchings where consumer and producer desires are partially already satisfied by local, outer matching and partially remain unsatisfied. The remaining desires also resolve congestion by dividing the available capacity.

Definition 5.2 (FairMax and FairBase Allocations). Consider a congestion tree $T=(V, E, A)$. Let $c(r)$ be the set of child vertices of the root $r$. For each child $i \in c(r)$, let FairMax $i$ and FairBase $i$ be the FairMax and FairBase allocations on $T_{i}=\left(V_{i}, E_{i}, A_{i}\right)$. Let the remaining desire Rem for agents $a \in A$ be defined as

$$
\operatorname{Rem}(a)= \begin{cases}\operatorname{FairMax}_{i}(a)-\text { FairBase }_{i}(a) & \text { if } a \in A_{i}, i \in c(r) \\ d_{a} & \text { if } a \in A \text { is at } r\end{cases}
$$


In the case that $\operatorname{Rem}(Q) \geq|\operatorname{Rem}(P)|$, define the FairMax allocation on the congestion tree $T=(V, E, A)$ as follows:

For a producer $p \in P_{i}$ with $i \in c(r)$,

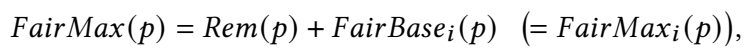

for a producer $p \in P$ at $r$,

$$
\operatorname{FairMax}(p)=\operatorname{Rem}(p)
$$

for a consumer $q \in Q_{i}$ with $i \in c(r)$,

$$
\operatorname{FairMax}_{(q)}=\operatorname{Div}(\text { Allowance, Rem, } Q)(q)+\text { FairBase }_{i}(q) \text {, }
$$

for a consumer $q \in Q$ at $r$,

$\operatorname{FairMax}(q)=\operatorname{Div}($ Allowance, Rem, $Q)(q)$,

where Allowance $=|\operatorname{Rem}(P)|+C_{r}$.

The definition of the FairBase allocation on the congestion tree $T=(V, E, A)$ is similar, taking instead Allowance $=|\operatorname{Rem}(P)|$.

In the case that $\operatorname{Rem}(Q) \leq|\operatorname{Rem}(P)|$ the definitions are analogous, only switching consumers and producers.

Informally, the definition of the FairMax allocation is as follows. The remaining desire Rem in Equation (7) represents the fact that some parts of the desires of consumers and producers are already matched with each other in the subtrees of the congestion tree $T=$ $(V, E, A)$. See also Figure 4 for a visual aide. If the root $r$ has no children, i.e. it is a leaf, then Equations (8) and (10) do not apply; this is the base case of the recursion. When the consumers outweigh the producers, the producer desires can be fully matched with consumer desires as seen in Equations (8) and (9). The consumers, however, have to divide the sum of producer desires: the Allowance. The FairMax allocation also includes interaction with the external network up to the capacity $C_{r}$ in the Allowance, on top of matching with the producer desires, as seen in Equations (10) and (11). Finally, of course, all agents in subtrees also get the desires that were already satisfied in those subtrees as seen in Equations (8) and (10).

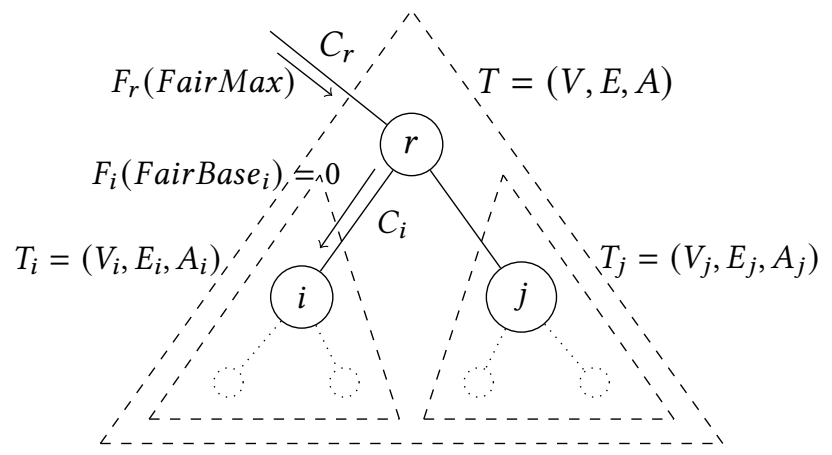

Figure 4: A representation of the recursive definition.

Proposition 5.3. The FairMax and FairBase allocations are, respectively, a max allocation and a base allocation.

Proof. The proof is by induction. Let $c(r)$, FairMax $_{i}$, FairBase $_{i}$, Rem, and Allowance be as in Definition 5.2.

For the induction basis, let $T=(V, E, A)$ be a congestion tree with only one vertex. Without loss of generality, assume that $\operatorname{Rem}(Q) \geq$
$|\operatorname{Rem}(P)|$. Note that $\operatorname{Rem}(a)=d_{a}$ for all agents $a \in A$. Since $D(a) \in[0, \operatorname{Rem}(a)]$ by Definition 4.1, it follows from Equations (9) and (11) and their analogs for FairBase that both FairMax and FairBase are desire compatible allocations. Moreover, it follows from Equation (11) and its analog for FairBase, respectively, that

$$
\begin{array}{ll}
\operatorname{FairMax}(S) \leq-\operatorname{FairMax}(P)+C_{r} & \Rightarrow \operatorname{FairMax}(A) \leq C_{r} \\
\operatorname{FairBase}(S)=-\operatorname{FairBase}(P) & \Rightarrow \operatorname{FairBase}(A)=0
\end{array}
$$

and thus that both FairMax and FairBase are congestion free allocations. Consequently, both FairMax and FairBase are feasible allocations.

Since by Equation (9) and its analog for FairBase,

$$
\operatorname{FairMax}(P)=\operatorname{FairBase}(P)=\sum_{p \in P} d_{p},
$$

it follows from Equation (13) that FairBase is a base allocation, and from the properties of the division in Equation (11) that FairMax is a max allocation.

For the induction step, let $T=(V, E, A)$ be a more general congestion tree, and assume that the proposition holds for subtrees $T_{i}=\left(V_{i}, E_{i}, A_{i}\right)$ with $i$ children of $r$. The proof of the induction step is similar to that of the induction basis. Again, without loss of generality, assume that $\operatorname{Rem}(Q) \geq|\operatorname{Rem}(P)|$. Since FairMax $i$ is a desire compatible allocation for all children $i$ of $r$, it follows in the same way as before from Definition 4.1, Equations (8) to (11) and their analogs for FairBase that both FairMax and FairBase are desire compatible allocations. Moreover, since $F_{i}\left(\right.$ FairBase $\left._{i}\right)=0$ for all children $i$ of $r$, it follows in the same way as before from Equations (10) and (11) and their analogs for FairBase, respectively, that

$$
\begin{array}{ll}
\operatorname{FairMax}(S) \leq-\operatorname{FairMax}(P)+C_{r} & \Rightarrow \operatorname{FairMax}(A) \leq C_{r} \\
\operatorname{FairBase}(S)=-\operatorname{FairBase}(P) & \Rightarrow \operatorname{FairBase}(A)=0
\end{array}
$$

and thus, since FairMax $i$ and FairBase $_{i}$ are congestion free allocations for all children $i$ of $r$, that both FairMax and FairBase are congestion free allocations. Consequently, both FairMax and FairBase are again feasible allocations.

Since by Equations (8) and (9) and their analogs for FairBase,

$$
\begin{aligned}
\operatorname{FairMax}(P) & =\text { FairBase }(P) \\
& =\sum_{i \text { child of } r} \sum_{p \in P_{i}} \operatorname{FairMax}_{i}(p)+\sum_{p \in P \text { at } r} d_{p},
\end{aligned}
$$

and FairMax $i$ is a max allocation for all children $i$ of $r$, it follows from Equation (16) that FairBase is a base allocation, and from the properties of the division in Equations (10) and (11) that FairMax is a max allocation.

Theorem 5.4. The FairMax and FairBase allocations are, respectively, a max allocation and a base allocation that are local, outer matchings.

Proof. Let $c(r)$ and FairBase $i$ be as in Definition 5.2.

The theorem follows from Proposition 5.3 and the fact, as seen from Equations (8) and (10) and their analogs for FairBase, that the restrictions of FairMax and FairBase to a subtree $T_{i}=\left(V_{i}, E_{i}, A_{i}\right)$ for a child $i \in c(r)$ fully contain the allocation FairBase $e_{i}$. Since 
FairBase $_{i}$ is a base allocation and thus maximizes the locally balanced flow on the subtree $T_{i}=\left(V_{i}, E_{i}, A_{i}\right)$, so do FairMax and FairBase.

Through induction on subtrees it then follows that the FairMax and FairBase allocations maximize the locally balanced flow on all subtrees of the congestion network $T=(V, E, A)$.

\section{ALGORITHM: LOCAL, OUTER MATCHING COMBINED WITH FAIR DIVISION}

This section presents an algorithmic mechanism that combines local, outer matching with notions of fair division to compute the FairMax and FairBase allocations on a congestion tree $T=$ $(V, E, A)$. A sketch of the approach is as follows. First compute these allocations on subtrees $T_{i}=\left(V_{i}, E_{i}, A_{i}\right)$ for vertices $i \in V$ from the leaves towards the root $r$. Each step considers one vertex $i$ and the subtree $T_{i}=\left(V_{i}, E_{i}, A_{i}\right)$. In this way, at each step, the FairMax and FairBase allocations for all subtrees with roots that are children of the current vertex will have been computed already.

Algorithm 1 uses the divide function from Algorithm 2 to fairly divide certain values over sets of agents. This is used for local, outer matching and for resolving any potential congestion. The divide function depends on the notion of fairness.

When the notion of fairness is egalitarian or nondiscriminatory, the divide function in Algorithm 2 uses the water level function from Algorithm 3. The water level function computes the level $w$ that an allocation on a set of agents must be reduced to, per agent, in order to divide a certain value equally over the set of agents.

\subsection{Algorithmic Local, Outer Matching}

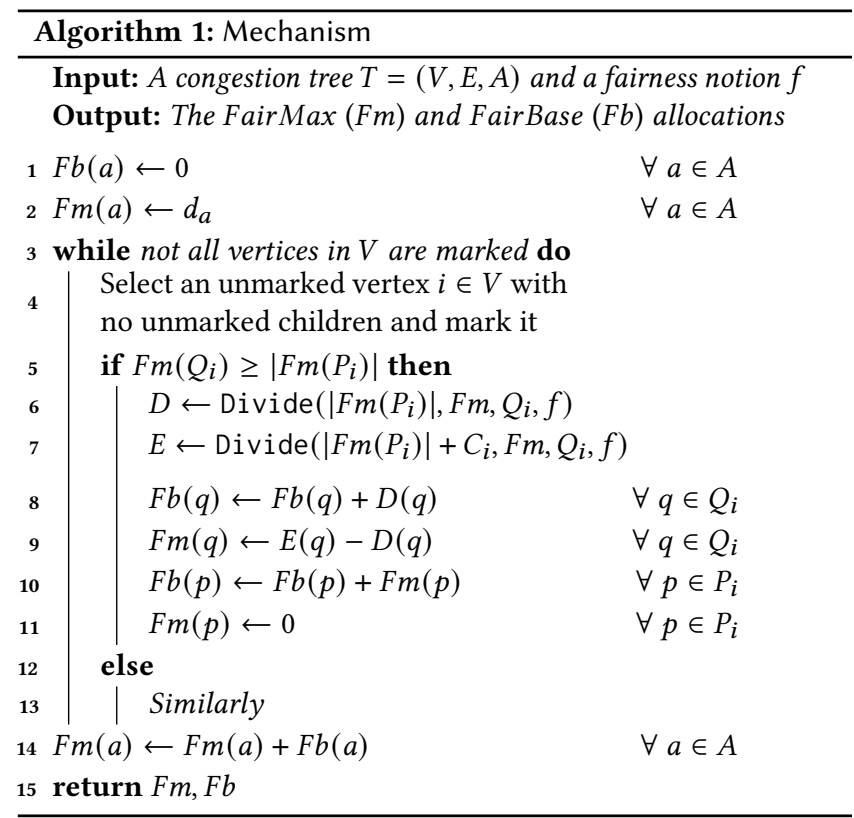

Algorithm 1 visits all vertices in $V$ exactly once, moving from the leaves towards the root $r$. At each step corresponding to a vertex $i$, for each agent $a \in A_{i}$, two variables are subject to change. The first variable, $F b(a)$, is from the FairBase allocation and can be thought of as the satisfied desire that has already been allocated to agent $a$ when considering only $T_{i}=\left(V_{i}, E_{i}, A_{i}\right)$. The second variable, $F m(a)$, can be thought of as the remaining desire that can still be allocated to agent $a$ in addition to its already satisfied desire, when considering only $T_{i}=\left(V_{i}, E_{i}, A_{i}\right)$.

At each step, the remaining desires $F m$ of the consumers and producers in $A_{i}$ are maximally matched with each other. This maximal matching is performed, when the consumers outweigh the producers, by dividing the sum of the remaining producer desires over the remaining consumer desires. The matched amounts are moved from the remaining desires $F m$ to the satisfied desires $F b$. This leaves either only consumers or only producers in terms of remaining desires $F m$ since either all consumers or all producers have their entire remaining desires $F m$ moved to their satisfied desires $F b$.

Simultaneously, at each step, any potential congestion is resolved by dividing the available capacity over the agents. This is done, when the consumers outweigh the producers, by dividing an amount equal to the sum of producer desires plus the capacity $C_{i}$ over the consumer desires. This amount can be thought of as the allowance of the consumers.

As noted before, the satisfied desires $F b$ will constitute the FairBase allocation. However, the remaining desires Fm do not yet constitute the FairMax allocation. In order to obtain the FairMax allocation, after all vertices have been visited by the algorithm, the remaining desires $F m$ and satisfied desires $F b$ are added together.

At this point it has become easy to see that Algorithm 1 indeed computes the FairMax and FairBase allocations. This result will be formalized in Section 6.4.

\subsection{Division for Different Notions of Fairness}

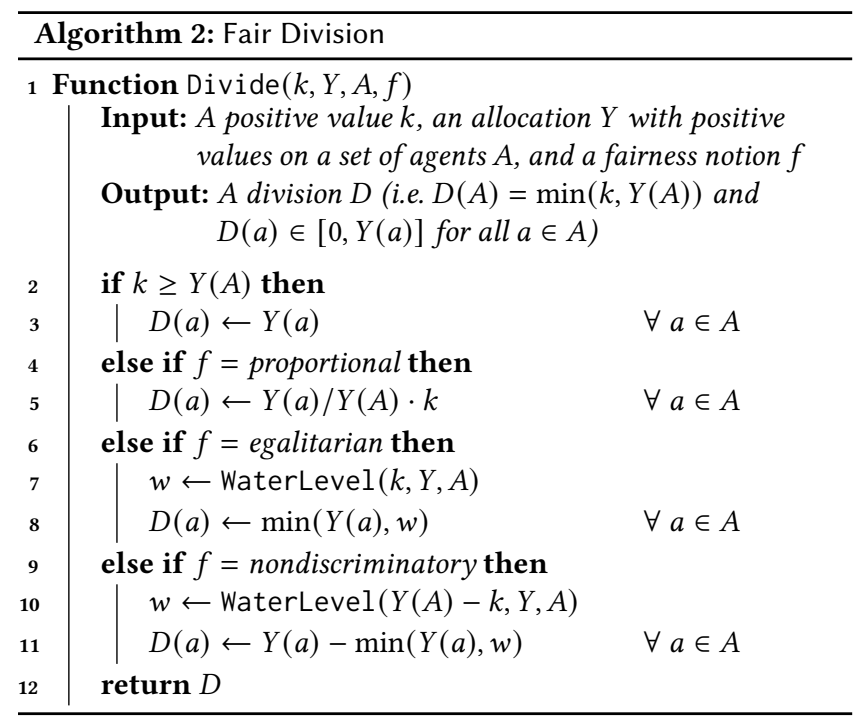

The division function in Algorithm 2 implements the division from Definition 4.1. If the value $k$ to divide is not larger than $Y(A)$, the function returns a division $D$ on $A$ that exactly divides the value $k$ over the agents $a \in A$ while not exceeding the claims $Y(a)$ for agents $a \in A$. 
The division function presented in Algorithm 2 supports the three principal notions of fair division discussed in Section 4 . However, it is of course possible to add any other possible division.

The division for the proportional notion of fairness is computed with a straightforward ratio multiplication, allocating to each agent $a \in A$ a portion of the value $k$ that is proportional to the ratio of the claim $Y(a)$ to the sum of claims $Y(A)$.

To compute the divisions for the egalitarian and nondiscriminatory notions of fairness, the concept of the water level is used by calling the water level function from Algorithm 3. These two divisions are computed by respectively reducing the claims $Y(a)$ to a water level $w$ and by reducing the claims $Y(a)$ by a water level $w$, to a minimum of zero.

For the proportional notion of fairness, the computed division $D$ trivially satisfies the output conditions. For the egalitarian notion of fairness, the computed division $D$ can directly been seen to satisfy the output conditions by considering the output condition of the water level function from Algorithm 3. To see that the computed division $D$ also satisfies the output conditions for the nondiscriminatory notion of fairness, consider that

$$
\sum_{a \in A} \min (Y(a), w)=Y(A)-k
$$

and thus that

$$
D(A)=Y(A)-\sum_{a \in A} \min (Y(a), w)=k .
$$

Informally, the part $Y(A)-k$ that will not be allocated is divided evenly over the agents $A$ and subtracted from their claims $Y(A)$.

\subsection{Setting the Water Level}

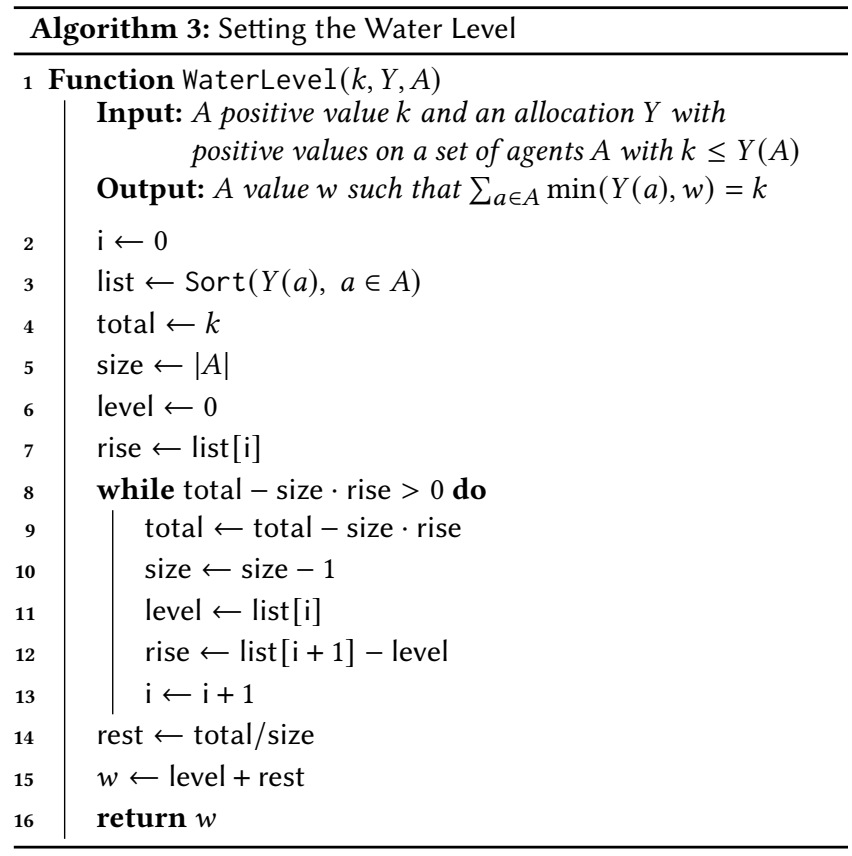

Setting the water level refers to uniformly dividing a good over claims by computing a single value referred to as the water level.
This water level value is used by egalitarian and nondiscriminatory notions of fair division.

The water level function in Algorithm 3 takes a value $k$ and an allocation $Y$ on a set of agents $A$. It then computes the unique level $w$ that the values $Y(a)$ for agents $a \in A$ must be reduced to in order to exactly divide the value $k$. Simply setting a single value ( $k$ divided by the number of agents in $A$ ) for all agents $a \in A$ does not reach the intended goal as some agents may have claims lower than that value. If that is the case, the unclaimed difference can be divided over the other agents.

To do this, the water level function in Algorithm 3 starts by sorting the claims $Y(a)$ from lowest to highest. It then checks if it can allocate the lowest claim to all agents $a \in A$. If yes, the lowest claim is removed. It then checks if it can also allocate the next lowest claim to all remaining agents. Once the next lowest claim cannot be allocated to all remaining agents, the rest of the unallocated value $k$ is evenly divided over the remaining agents.

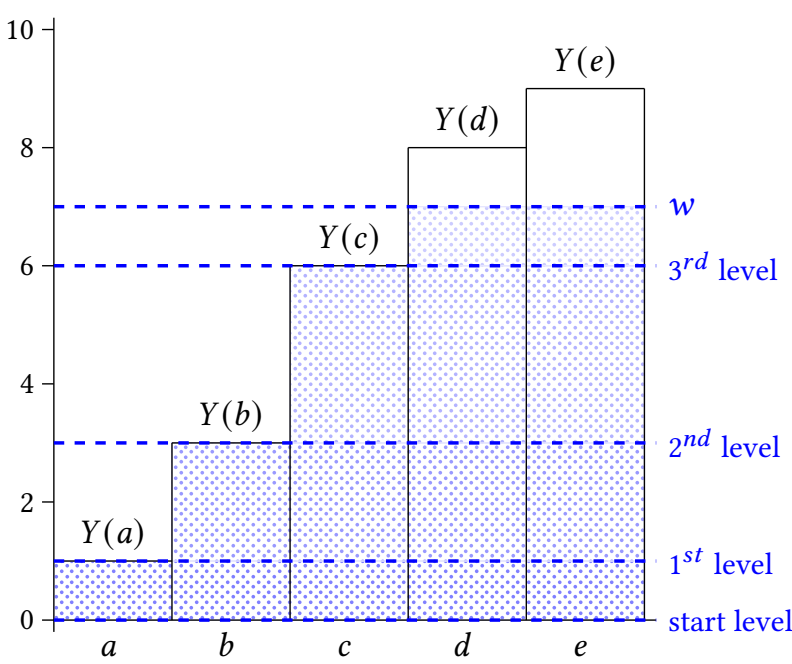

Figure 5: Steps of the water level function from Algorithm 3, dividing the value $k=24$ (hatched surface area) over five agents $a$ through $e$ with respective claims $1,3,6,8$, and 9 .

The name of the function comes from this repeated raising of the allocation from claim to claim that resembles the rising of a water level. Like water poured into a series of connected containers, it divides the quantity equally over the recipients. See also Figure 5.

\subsection{Result of the Algorithm}

TheOREm 6.1. Algorithm 1 computes the combination of local, outer matching with principal notions of fair division, resulting in the FairMax and FairBase allocations that correspond to maximal or no interaction with the external grid, respectively.

Proof. At each step of Algorithm 1, the remaining desire $F m$ is equal to the remaining desire allocation Rem from Definition 5.2. Instead of adding the $F b$ values and subtracting them again in the next step, they are saved and added to the $F m$ values only at the 
end of the algorithm. The variable updates in the algorithm are identical to those in Definition 5.2.

The division function used in Algorithm 1 is provided by Algorithm 2, and allows for combination with any of the three discussed principal notions of fair division.

\subsection{Example Congestion Solutions}

Figure 6 revisits the simple example congestion tree from Figure 3.

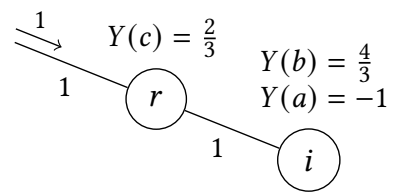

Proportional FairMax

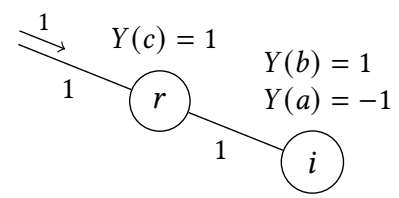

Nondiscriminatory FairMax

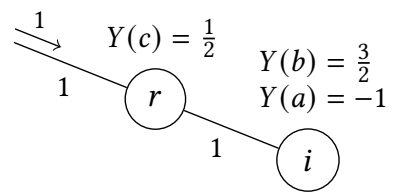

Egalitarian FairMax

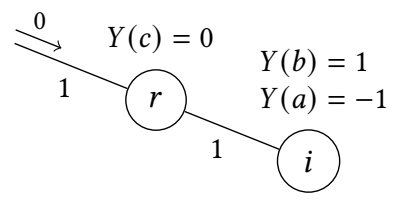

FairBase
Figure 6: FairMax allocations with different notions of fair division for the simple congestion network $T=(V, E, A)$ from Figure 3. In this example, the FairBase allocation is the same for all three principal notions of fair division.

Figure 7 shows a more complex congestion tree for which the FairMax allocation is computed with the egalitarian notion of fair division. Figure 8 shows the steps that Algorithm 1 takes during this computation. Once the root $r$ is reached, the FairMax allocation is computed by adding the $F b$ values to the $F m$ values.

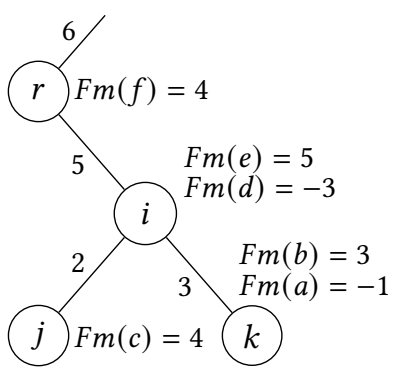

Initial Fm

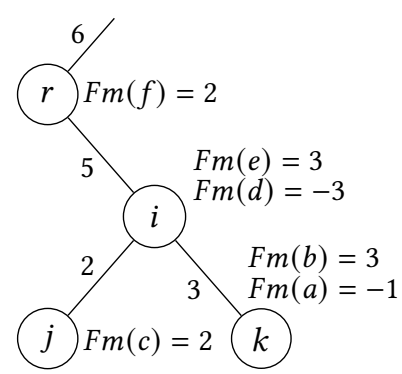

Egalitarian FairMax
Figure 7: Algorithm input (left) and output (right).

\section{INCENTIVE COMPATIBILITY}

One of the central concepts in the field of mechanism design is that of incentive compatibility. A mechanism is designed with a certain outcome in mind, for example a congestion free power flow network. The designer of a mechanism lays out the rules in such a way that

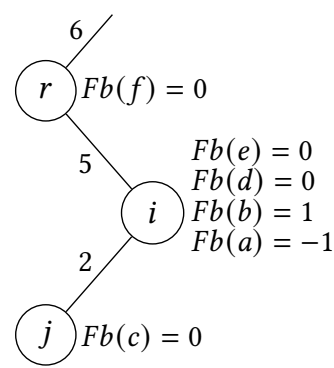

$F b$ after $k$

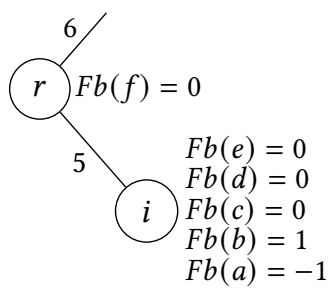

$F b$ after $j$

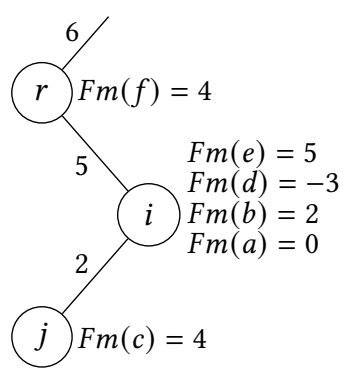

$F m$ after $k$

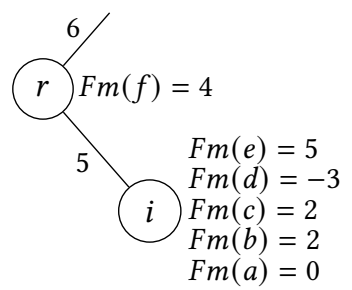

$F m$ after $j$

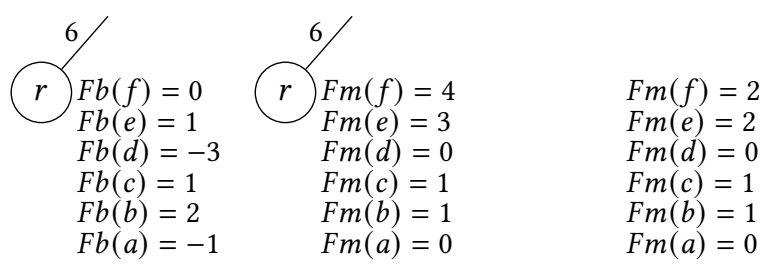

$F b$ after $i$ (and $r$ ) Fm after $i$

Fm after $r$
Figure 8: Algorithm steps from vertex $k$ to $r$.

agents playing the game follow strategies that together reach the intended outcome. This assumes that the agents want to play the game, and that they will play it honestly. An incentive compatible mechanism ensures that agents will participate truthfully.

The importance of truthful participation is emphasized when dealing with fairness. Fairness often heavily depends on the outcomes for the agents relative to each other. If agents can 'game the system', the agents that do will obtain an unfair advantage over the other agents. A mechanism that allows such strategies will require strong assumptions about the participating agents or other means of enforcing fairness.

Proposition 7.1. The mechanism presented in Algorithm 1 is incentive compatible when the notion of fairness is egalitarian.

Proof. Consider the $F m$ output of the mechanism, the proof for the $F b$ output is analogous. Let $T=(V, E, A)$ be a congestion tree containing an agent $a$ with true desire $d_{a}^{*}$. Without loss of generality, assume that agent $a$ is a consumer, i.e. $d_{a}^{*}>0$.

If agent $a$ reports $d_{a}=d_{a}^{*}$ and the mechanism returns $F m(a)=$ $d_{a}^{*}$, then the agent cannot improve its situation by reporting another desire $d_{a}$ because it is already in its preferred situation. 
If agent $a$ reports $d_{a}=d_{a}^{*}$ and the mechanism returns $F m(a)<$ $d_{a}^{*}$, then the agent may try reporting another desire $d_{a} \neq d_{a}^{*}$ to improve its situation. Since the mechanism returned $F m(a)<d_{a}$ when reporting $d_{a}=d_{a}^{*}$, it must be that the value of $F m(a)+F b(a)$ was reduced at at least one step of the algorithm. Consider the first step at which this happened, and the vertex $i$ corresponding to that step. It must be that, initially, congestion occurred at this vertex, i.e. $\left|F m\left(P_{i}\right)\right|+C_{i}<F m\left(Q_{i}\right)$ at Algorithm 1 Line 7. Since $F m(a)+F b(a)$ was reduced at this vertex, it must have been true that $F b(a)>w$ at Algorithm 2 Line 8. Note that the output $w$ of the water level function in Algorithm 3 does not depend on values $Y(a)>w$.

It now follows that reporting $d_{a}>d_{a}^{*}$ causes the same congestion at vertex $i$, i.e. $\left|F m\left(P_{i}\right)\right|+C_{i}<F m\left(Q_{i}\right)$ at Algorithm 1 Line 7, and produces the same value $w$ at Algorithm 2 Line 7, resulting in the same reduction of $F m(a)+F b(a)$ at this step as compared to reporting $d_{a}=d_{a}^{*}$. Thus after this step, there is no difference between reporting $d_{a}=d_{a}^{*}$ and reporting $d_{a}>d_{a}^{*}$, leading the mechanism to return the exact same value $F m(a)<d_{a}^{*}$ in both cases.

It is possible that reporting $d_{a}>d_{a}^{*}$ causes congestion at an earlier step corresponding to a vertex $j$. This does not change the argument, since reporting $d_{a}=d_{a}^{*}$ not causing congestion at vertex $j$ implies that when reporting $d_{a}>d_{a}^{*}, F m(a)+F b(a)$ can at most be reduced to $d_{a}^{*}$ at vertex $j$.

Alternatively, reporting $d_{a}<d_{a}^{*}$ may avoid the congestion at vertex $i$. However, this is only the case when $\operatorname{Fm}(a)$ at Algorithm 1 Line 7 is equal to or lower than the value $w$ at Algorithm 2 Line 7 when reporting $d_{a}=d_{a}^{*}$. In other words, the reduction of $F m(a)+F b(a)$ to $w+F b(a)$ at vertex $i$ is only avoided if it is already equal to or lower than $w+F b(a)$. The same argument then applies to each following step corresponding to a vertex $j$ with $a \in A_{j}$ where congestion occurs when reporting $d_{a}=d_{a}^{*}$. It follows that the mechanism output $F m(a)$ when reporting $d_{a}<d_{a}^{*}$ is equal to or lower than the output $\operatorname{Fm}(a)$ when reporting $d_{a}=d_{a}^{*}$.

This shows that agent $a$ cannot improve its situation by reporting anything other than its true desire $d_{a}^{*}$, i.e., being truthful is a weakly dominant strategy.

Proposition 7.2. The mechanism presented in Algorithm 1 is not incentive compatible when the notion of fairness is proportional or nondiscriminatory.

Proof. The proof is by counterexample. Let $T=(V, E, A)$ be a simple congestion tree with only one vertex $r$, a capacity of $C_{r}=8$ on its virtual edge, and two agents $a$ and $b$ with true desires $d_{a}^{*}=6$ and $d_{b}^{*}=6$. If both agents report their true desires, the mechanism would return $F m(a)=4$ and $F m(b)=4$ for both the proportional and the nondiscriminatory notions of fairness. However, if agent $a$ instead reports $d_{a}=10$ while agent $b$ still reports its true desire $d_{b}=6$, then the mechanism would return $F m(a)=5$ and $F m(b)=3$ for the proportional notion of fairness, or $F m(a)=6$ and $F m(b)=$ 2 for the nondiscriminatory notion of fairness. This shows that agent $a$ can improve its situation by reporting a desire designed for participation in the mechanism, rather than its true desire.

The same counterexample can be used for the $F b$ output of the mechanism by adding a third agent $c$ with $d_{c}^{*}=-8$.
Propositions 7.1 and 7.2 can be intuitively understood to hold true by examining Figure 2 . The three divisions corresponding to the three principal notions of fair division are found at the intersection of their respective lines with the line representing the quantity of the good to be divided.

Two of the three principal notions of fair division correspond to lines that depend on the point of the claims, and thus the point of intersection depends on the point of the claims as well. Evidently, for these two notions, the division correlates directly with the claims reported by the agents. Indeed, for the proportional and nondiscriminatory notions of fairness, an agent can directly influence the division.

Conversely, for the egalitarian notion of fairness, the point of intersection only depends on claims to a limited extent. Only an agent that is allocated its entire claim would potentially be allocated a larger amount by reporting a higher claim. Agents with claims above a certain threshold cannot increase the amount allocated to them by reporting a higher claim. The egalitarian division therefore only correlates with the claims reported by the agents to the point that each agent can claim their fair equal share and receive it.

\section{COMPUTATIONAL COMPLEXITY}

The water level function in Algorithm 3 sorts the $Y(a)$ values in $O(m \cdot \log (m))$ time, where $m$ is the number of agents in the input set $A$. It then enters a while loop which takes at most $m-1$ iterations. Thus the computational complexity of the water level function in Algorithm 3 is $O(m \cdot \log (m))$.

Algorithm 1 visits each vertex in $V$ exactly once, and at each vertex calls the divide function in Algorithm 2 two times. For the proportional notion of fairness, the divide function assigns $m$ values, where $m$ is the number of agents in the input set $A$. For the egalitarian and nondiscriminatory notions of fairness, the divide function calls the water level function at most once.

Therefore, the worst case computational complexity of Algorithm 1 with the proportional notion of fairness is $O(n \cdot m)$, while with the egalitarian and nondiscriminatory notions of fairness it is $O(n \cdot m \cdot \log (m))$. Here $n$ is the number of vertices in $V$ and $m$ is the total number of agents in $A$.

\section{CONCLUSION AND DISCUSSION}

We presented local, outer matching, Definition 5.1, as a novel concept of fairness for congestion management in low-voltage networks. Local, outer matching addresses the important problem of fairness in matching by prioritizing local matching in the peripheral where prosumers are furthest away from the substation. We then presented congestion solutions in Definition 5.2 that were proven by Theorem 5.4 to be local, outer matchings that make maximal use of the available network capacity. These congestion solutions interchangeably employ established notions of fair division for dividing quantities such as capacity. In Section 4 we discussed three distinct principal notions of fair division: proportional, egalitarian, and nondiscriminatory division.

Subsequently, in Section 6, we presented an algorithmic mechanism that combines local, outer matching with notions of fair division and computes congestion solutions which fairly resolve congestion and make maximal use of available network capacity as 
proven by Theorem 6.1. In Section 6.2 we showed that the mechanism is able to employ different notions of fair division, and we then went on to prove that the egalitarian notion of fairness results in an incentive compatible mechanism, Proposition 7.1, while the proportional and nondiscriminatory notions of fairness do not, Proposition 7.2.

Finally, in Section 8, we showed that the presented congestion solutions can be computed by an algorithm with low computational complexity. This makes the notion of fairness and the algorithm suitable for sizeable and time sensitive congestion problems such as those encountered in electrical grids.

The egalitarian notion of fair division resulting in an incentive compatible mechanism is an obvious advantage over other notions of fair division, but does not render other notions of fair division obsolete. Consensus on the accepted notion of fair division should be a priority since fairness is fundamentally subjective and dependent on setting. Additional penalties or incentives could be implemented to make other notions of fair division feasible for use in this setting if they are strongly preferred.

The algorithmic mechanism we presented in this paper is limited to the acyclic networks that are found in real-world low-voltage networks. It is likely that a similar algorithmic mechanism for more general network structures would have a higher computational complexity. Running in limited computational time is, however, essential for the application in this domain.

The theoretical foundation that this paper lays may be extended to more detailed models, for example including line losses by discounting flows per line that is traversed. This raises the interesting question of whether fairness lies with the sent quantity or the received quantity.

Another potential avenue of research would be to look at other fair ways of matching consumer and producer desires, for example by changing the hierarchical structure of matching or by introducing time-shiftable consumers and producers.

\section{ACKNOWLEDGMENTS}

This work is part of the research programme SES-BE with project number 14182 P13-21, which is partly financed by the Dutch Research Council (NWO).

\section{REFERENCES}

[1] Rediet Abebe, Jon Kleinberg, and David C. Parkes. 2017. Fair Division via Social Comparison. In Proceedings of the 16th Conference on Autonomous Agents and MultiAgent Systems (São Paulo, Brazil) (AAMAS '17). International Foundation for Autonomous Agents and Multiagent Systems, Richland, SC, 281-289.

[2] Noga Alon. 1987. Splitting necklaces. Advances in Mathematics 63, 3 (1987), 247 253. https://doi.org/10.1016/0001-8708(87)90055-7

[3] Haris Aziz and Simon Mackenzie. 2016. A Discrete and Bounded Envy-Free Cake Cutting Protocol for Four Agents. In Proceedings of the Forty-Eighth Annual ACM Symposium on Theory of Computing (Cambridge, MA, USA) (STOC '16). Association for Computing Machinery, New York, NY, USA, 454-464. https: //doi.org/10.1145/2897518.2897522

[4] P. Bach Andersen, J. Hu, and K. Heussen. 2012. Coordination strategies for distribution grid congestion management in a multi-actor, multi-objective setting. In 2012 3rd IEEE PES Innovative Smart Grid Technologies Europe (ISGT Europe). 1-8.

[5] Steven J. Brams and Alan D. Taylor. 1995. An Envy-Free Cake Division Protocol The American Mathematical Monthly 102, 1 (1995), 9-18. https://doi.org/10.1080/ 00029890.1995 .11990526

[6] R. Ciavarella, M. Di Somma, G. Graditi, and M. Valenti. 2019. Congestion Management in distribution grid networks through active power control of flexible distributed energy resources. In 2019 IEEE Milan PowerTech. 1-6. https:
//doi.org/10.1109/PTC.2019.8810585

[7] Dominik Danner, Wolfgang Duschl, and Hermann de Meer. 2019. Fair Charging Service Allocation for Electric Vehicles in the Power Distribution Grid. In Proceedings of the Tenth ACM International Conference on Future Energy Systems (Phoenix, AZ, USA) (e-Energy '19). Association for Computing Machinery, New York, NY, USA, 406-408. https://doi.org/10.1145/3307772.3330168

[8] L. E. Dubins and E. H. Spanier. 1961. How to Cut a Cake Fairly. The American Mathematical Monthly 68, 1P1 (1961), 1-17. https://doi.org/10.1080/00029890. 1961.11989615

[9] European Parliament. 2016. Clean Energy For All Europeans

[10] G. Gamow and M. Stern. 1958. Puzzle-math. Viking Press.

[11] Avital Gutman and Noam Nisan. 2012. Fair Allocation without Trade. In Proceedings of the 11th International Conference on Autonomous Agents and Multiagent Systems - Volume 2 (Valencia, Spain) (AAMAS '12). International Foundation for Autonomous Agents and Multiagent Systems, Richland, SC, 719-728.

[12] A.N.M.M. Haque, P.H. Nguyen, F.W. Bliek, and J.G. Slootweg. 2017. Demand response for real-time congestion management incorporating dynamic thermal overloading cost. Sustainable Energy, Grids and Networks 10 (2017), 65 - 74. https://doi.org/10.1016/j.segan.2017.03.002

[13] Brinn Hekkelman and Han La Poutré. 2019. Fairness in Smart Grid Congestion Management. In 2019 IEEE PES Innovative Smart Grid Technologies Europe (ISGTEurope). IEEE, 1-5. https://doi.org/10.1109/ISGTEurope.2019.8905496

[14] Junjie Hu, Arshad Saleem, Shi You, Lars Nordström, Morten Lind, and Jacob Østergaard. 2015. A multi-agent system for distribution grid congestion management with electric vehicles. Engineering Applications of Artificial Intelligence 38 (2015), 45 - 58. https://doi.org/10.1016/j.engappai.2014.10.017

[15] J. Hu, S. You, M. Lind, and J. Østergaard. 2014. Coordinated Charging of Electric Vehicles for Congestion Prevention in the Distribution Grid. IEEE Transactions on Smart Grid 5, 2 (2014), 703-711.

[16] Ali Khodabakhsh, Jimmy Horn, Evdokia Nikolova, and Emmanouil Pountourakis. 2019. Prosumer Pricing, Incentives and Fairness. In Proceedings of the Tenth ACM International Conference on Future Energy Systems (Phoenix, AZ, USA) (eEnergy '19). Association for Computing Machinery, New York, NY, USA, 116-120. https://doi.org/10.1145/3307772.3328304

[17] Ruoyang Li, Qiuwei Wu, and Shmuel S. Oren. 2014. Distribution Locational Marginal Pricing for Optimal Electric Vehicle Charging Management. IEEE Transactions on Power Systems 29, 1 (07 2014), 203-211.

[18] Christian Nabe, Marie-Louise Arlt, Michael Döring, Uwe Holzhammer, and Norman Gerhardt. 2017. Smart-Market-Design in Deutschen Verteilnetzen. Technical Report. Ecofys and Fraunhofer IWES, requested by Agora Energiewende.

[19] Elisha A. Pazner and David Schmeidler. 1978. Egalitarian Equivalent Allocations: A New Concept of Economic Equity*. The Quarterly fournal of Economics 92, 4 (11 1978), 671-687. https://doi.org/10.2307/1883182

[20] R.M. Philipsen, M.M. de Weerdt, and L.J. de Vries. 2016. Auctions for Congestion Management in Distribution Grids. 13th International Conference on the European Energy Market (2016), 1-5. https://doi.org/10.1109/EEM.2016.7521326

[21] Jose Rivera, Christoph Goebel, and Hans-Arno Jacobsen. 2015. A Distributed Anytime Algorithm for Real-Time EV Charging Congestion Control. In Proceedings of the 2015 ACM Sixth International Conference on Future Energy Systems (Bangalore, India) (e-Energy '15). Association for Computing Machinery, New York, NY, USA, 67-76. https://doi.org/10.1145/2768510.2768544

[22] C. Shao, X. Wang, M. Shahidehpour, X. Wang, and B. Wang. 2017. Partial Decomposition for Distributed Electric Vehicle Charging Control Considering Electric Power Grid Congestion. IEEE Transactions on Smart Grid 8, 1 (Jan 2017), 75-83. https://doi.org/10.1109/TSG.2016.2595494

[23] Hugo Steinhaus. 1948. Report of the Washington Meeting, September 6-18, 1947. Econometrica 16, 1 (1948), 101-104. http://www.jstor.org/stable/1914289

[24] Walter Stromquist. 1980. How to Cut a Cake Fairly. The American Mathematical Monthly 87, 8 (1980), 640-644. https://doi.org/10.1080/00029890.1980.11995109

[25] Stijn Vandael, Nelis Boucké, Tom Holvoet, Klaas De Craemer, and Geert Deconinck. 2011. Decentralized Coordination of Plug-in Hybrid Vehicles for Imbalance Reduction in a Smart Grid. In The 10th International Conference on Autonomous Agents and Multiagent Systems - Volume 2 (Taipei, Taiwan) (AAMAS '11). International Foundation for Autonomous Agents and Multiagent Systems, Richland, SC, $803-810$

[26] Remco A. Verzijlbergh, L.J. Vries, and Z Lukszo. 2014. Renewable Energy Sources and Responsive Demand. Do We Need Congestion Management in the Distribution Grid? IEEE Transactions on Power Systems 29, 5 (09 2014), 2119-2128.

[27] Perukrishnen Vytelingum, Sarvapali D. Ramchurn, Thomas D. Voice, Alex Rogers, and Nicholas R. Jennings. 2010. Trading Agents for the Smart Electricity Grid. In Proceedings of the 9th International Conference on Autonomous Agents and Multiagent Systems: Volume 1 - Volume 1 (Toronto, Canada) (AAMAS '10). International Foundation for Autonomous Agents and Multiagent Systems, Richland, SC, 897-904

[28] Zhenyu Xue, D. Z. Fang, Wen Sun, and Shiqiang Yuan. 2009. A Novel Approach for Dynamic Congestion Management in Open Power Markets. Proceedings of the 2009 International Conference on Energy and Environment Technology - Volume 02, 11-14. https://doi.org/10.1109/ICEET.2009.239 